\title{
Photo-assisted current and shot noise in the fractional quantum Hall effect
}

\author{
Adeline Crépieux ${ }^{a}$, Pierre Devillard ${ }^{b}$, and Thierry Martin $^{a}$ \\ ${ }^{a}$ Centre de Physique Théorique, Université de la Méditerranée, Case 90\%, 13288 Marseille, France \\ ${ }^{b}$ Centre de Physique Théorique, Université de Provence, Case 90\%, 13288 Marseille, France
}

\begin{abstract}
The effect of an AC perturbation on the shot noise of a fractional quantum Hall fluid is studied both in the weak and the strong backscattering regimes. It is known that the zero-frequency current is linear in the bias voltage, while the noise derivative exhibits steps as a function of bias. In contrast, at Laughlin fractions, the backscattering current and the backscattering noise both exhibit evenly spaced singularities, which are reminiscent of the tunneling density of states singularities for quasiparticles. The spacing is determined by the quasiparticle charge $\nu e$ and the ratio of the DC bias with respect to the drive frequency. Photo-assisted transport can thus be considered as a probe for effective charges at such filling factors, and could be used in the study of more complicated fractions of the Hall effect. A non-perturbative method for studying photo-assisted transport at $\nu=1 / 2$ is developed, using a refermionization procedure.
\end{abstract}

PACS numbers: 03.65.Ta, 03.67.Lx, 85.35.Be, 73.23.Ad

\section{INTRODUCTION}

In mesoscopic systems, the measurement of current and noise makes it possible to probe the effective charges which flow in conductors, and opens the possibility for studying the role of the statistics in stationary quantum transport experiments. This has been illustrated experimentally and theoretically in both cases where the interaction between

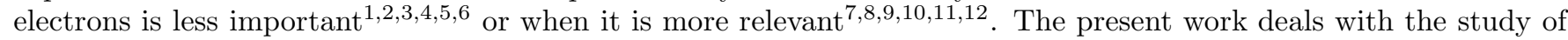
photo-assisted shot noise in a specific one dimensional correlated system: a Hall bar in the fractional Hall regime, for which charge transport occurs via two counter-propagating chiral edges states.

Over the years, the attention has been focusing also on the transport properties of systems on which an external harmonic perturbation is acting. A fundamental step is first to study the current response. For instance, it is possible to generate a DC current by applying voltage gates on which an AC perturbation is acting 13,14 . Here we consider the superposition of a DC bias with a time dependent perturbation. For normal and superconducting systems, it has been shown ${ }^{15.16}$ that the photo-assisted shot noise allows to retrieve information on the finite frequency noise, which was computed for ballistic systems $\frac{17}{7}$, and measured in diffusive metallic wires $\frac{18}{}$. Such finite frequency measurements turn out to be challenging in practice. On the other hand, if an experiment is devoted to zero frequency noise, information on the finite frequency noise spectrum can be retrieved provided that the system has an added, external, finite frequency perturbation. In particular, the presence of a small, additional harmonic perturbation modifies the phase of the charge carriers (reflection/transmission probabilities are affected to a lesser extent), and in some sense it acts as a probe to study the finite frequency noise spectrum of the conductor.

Note that the external modulation can either be imposed on a gate voltage, located in the vicinity of the conductor, which controls the transparency of the barrier. This corresponds to the Gedanken experiment for the traversal time in tunneling $\frac{19}{19}$ and to the early proposals for photo-assisted shot noise ${ }^{15.20}$. At the same time, the transport properties of an irradiated point contact ${ }^{21}$ have also been studied with this point of view. Alternatively, the modulation can be imposed from the leads to which the system is connected, which may be simpler to achieve in experiments, because no additional gating is required. For non-interacting electrons, it has been shown ${ }^{15}$ that the derivative of the current noise with respect to the bias exhibits evenly spaced steps whose height is specified by Bessel functions. This result - which was derived using scattering theory - has been generalized to diffusive metallic wires using known random matrix theory results and has been tested experimentally for normal diffusive metals 20 and recently for ballistic samples in a point contact geometry 22 .

One then enquires whether electronic correlations will play an important role on the photo-assisted shot noise characteristics. In multichannel conductors described by a scattering theory, transport properties (current and noise) have been analyzed while taking into account screening in a self-consistent treatment ${ }^{23}$. For a normal metalsuperconducting junction biased in the Andreev regime, the correlations in the superconductor are responsible for a doubling of the electron charge in the shot noise $\frac{16}{\underline{16}}$. The noise derivative with respect to bias voltage then exhibits steps whose spacing contains the charge of a Cooper pair, as confirmed by recent experiments 24.25 . Nevertheless, one can argue that NS systems are not so far from free electron systems, as these can be modeled by a scattering theory in which electrons are converted in hole and vice versa ${ }^{26}$.

Another possible ground for studying the effect of interactions is to consider a one-dimensional correlated electron system - a Luttinger liquid. Reduced dimensionality is known to affect drastically the current-voltage characteristics 27. 
The finite frequency current response of a one dimensional wire has been discussed in Ref. 28, while the effect of a localized time dependent perturbation on the conductance of a non-chiral Luttinger liquid was addressed recently in Ref. 29. The noise spectrum of a Hall bar in the fractional Hall regime was presented in Ref. 30. Here we choose the same model as the latter, mainly a simple fractional quantum Hall fluid consisting of two edge states with a point contact. The potential difference between the two edges has both a DC and an AC component. The time average current flowing in this system has been discussed using a semi-classical approach in Ref. 31. Here, we concentrate on the shot noise in both the weak and strong backscattering regimes. It is expected that the step like behavior of non interacting electrons will be strongly modified when the electron filling factor $\nu$ deviates from 1 . It is also expected that in the weak backscattering regime, the fractional charge $e^{*}=\nu e$ probed by DC shot noise experiments $8,9,10,11,32$ should appear explicitly: the spacing between singularities in the shot noise should be given by the Josephson frequency $e^{*} V_{0} / \hbar$, where $V_{0}$ is the DC bias voltage. Yet this remains to be shown in a first principles calculation.

The paper is organized as follows. The model Hamiltonian is presented in the next section, and the weak backscattering limit is considered in Sec. 3. Results for the strong backscattering limit are collected in Sec. 4 . Sec. 5 deals with the results using the refermionization procedure at filling factor $\nu=1 / 2$.

\section{MODEL HAMILTONIAN}

Consider first a fractional quantum Hall bar. The right and left moving chiral excitations are described by the Hamiltonian 33 :

$$
H_{0}=\left(v_{F} \hbar / 4 \pi\right) \sum_{r} \int d s\left(\partial_{s} \phi_{r}\right)^{2},
$$

with $r=R, L$ for right and left movers.

We adopt the simple, intuitive picture where depending on the strength of the impurity, either quasiparticles tunnel through the (single) quantum Hall fluid, or the impurity is so strong that the fluid is split into two, and only electrons can tunnel from one fluid to the other ${ }^{32}$. This picture has been sucessful in explaining the main features of transport in both regimes for the quantum Hall bar ${ }^{10,11}$. In the case of a weak impurity, the backscattering of quasiparticles is described by the tunneling Hamitonian:

$$
H_{B}(t)=\sum_{\varepsilon} A^{(\varepsilon)}(t)\left[\Psi_{R}^{\dagger}(t) \Psi_{L}(t)\right]^{(\varepsilon)},
$$

where the notation $\epsilon$ specifies the operator $(\epsilon=+)$ or its hermitian (complex) conjugate $(\epsilon=-)$ as in Ref. 34 . Let $\Gamma_{0}$ be the bare tunneling amplitude, and $V(t)=V_{0}+V_{1} \cos (\omega t)$ be the total (DC and AC) potential drop at the junction. Here, one has the choice of either including this voltage in the properties of the fractional edges, or to take this voltage into account using a gauge transformation. Indeed, one can choose a gauge where the electric field at the junction is fully specified by a vector potential only. This procedure is called the Peierls substitution. The quasiparticle field operator then acquires a phase which is tied to the gauge transformation function $\chi(t)=-c \int V(t) d t$. The corresponding hopping amplitude thus becomes:

$$
A(t)=\Gamma_{0} e^{-i e^{*} \chi(t) / \hbar c} .
$$

Note that the charge which appears in the phase factor is the fractional charge $e^{*}=\nu e$ corresponding to edge state quasiparticles. This choice is justified in the weak backscattering regime, where quasiparticles tunnel through the quantum Hall fluid. In addition, from the DC and AC voltage amplitudes, it is convenient to introduce new frequencies which include this anomalous charge:

$$
\omega_{0} \equiv e^{*} V_{0} / \hbar, \quad \omega_{1} \equiv e^{*} V_{1} / \hbar
$$

With this choice, the phase factor of the tunneling amplitude reads $e^{*} \chi(t) / \hbar c=-\left(\omega_{0} t+\left(\omega_{1} / \omega\right) \sin (\omega t)\right)$. In Eq. (2), $\Psi_{r}$ is the quasiparticle field associated to the left $(r=L)$ or right $(r=R)$ movers. It can be expressed in term of the bosonic chiral field $\phi_{r}$ :

$$
\Psi_{r}(t)=\frac{M_{r}}{\sqrt{2 \pi a}} e^{i \sqrt{\nu} \phi_{r}(t)},
$$

where $M_{r}$ is the Klein factor, which does not play any role in this lowest order calculation (because $M_{r}^{2}=1$ ), and $a$ is the short distance cutoff. 


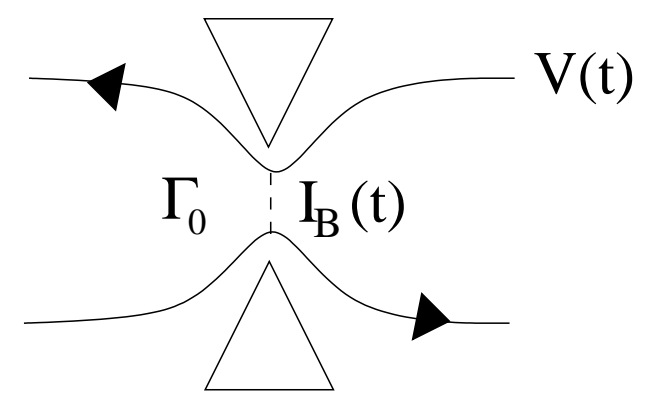

FIG. 1: Backscattering between edge states in presence of a bias voltage modulation.

\section{WEAK BACKSCATTERING LIMIT}

\section{A. Current}

The backscattering current can be calculated using $I_{B}(t)=-c \partial H_{B}(t) / \partial \chi(t)$. The calculation method of the current follows closely that of Refs. 8,12 34. For the voltage modulation, this means that the current operator reads:

$$
I_{B}(t)=\frac{i e^{*}}{\hbar} \sum_{\varepsilon} \varepsilon A^{(\varepsilon)}(t)\left[\Psi_{R}^{\dagger}(t) \Psi_{L}(t)\right]^{(\varepsilon)}
$$

for simplicity we set $\hbar=1$. Using the Keldysh formulation of non equilibrium transport, the average current is expressed as a time ordered contour:

$$
\left\langle I_{B}(t)\right\rangle=\frac{1}{2} \sum_{\eta}\left\langle T_{K}\left\{I_{B}\left(t^{\eta}\right) e^{-i \int_{K} d t_{1} H_{B}\left(t_{1}\right)}\right\}\right\rangle,
$$

where $\eta$ is the Keldysh index and the notation $K$ refers to the Keldysh contour. Expanding to lowest order in $\Gamma_{0}$ and taking into account quasiparticle conservation, the bosonized expressions for the quasiparticle fields are employed, together with the definition of the chiral Green's function ${ }^{8.35}$ :

$$
G^{\eta \eta^{\prime}}\left(t, t^{\prime}\right)=\left\langle T_{K}\left\{\phi_{r}\left(t^{\eta}\right) \phi_{r}\left(t^{\prime \eta^{\prime}}\right)\right\}\right\rangle-\frac{1}{2}\left\langle T_{K}\left\{\phi_{r}\left(t^{\eta}\right)^{2}\right\}\right\rangle-\frac{1}{2}\left\langle T_{K}\left\{\phi_{r}\left(t^{\prime \eta^{\prime}}\right)^{2}\right\}\right\rangle
$$

and the backscattering current associated with an (arbitrary) time dependent perturbation becomes:

$$
\left\langle I_{B}(t)\right\rangle=\frac{e^{*}}{8 \pi^{2} a^{2}} \sum_{\eta \eta_{1}} \eta_{1} \int_{-\infty}^{+\infty} d t_{1} e^{2 \nu G^{\eta \eta_{1}}\left(t, t_{1}\right)}\left(A(t) A^{*}\left(t_{1}\right)-A^{*}(t) A\left(t_{1}\right)\right) .
$$

Assume now that the bias voltage is modulated by a harmonic perturbation. Using the generating function of the Bessel function as in Ref. 19, $\exp \left[i\left(\omega_{1} / \omega\right) \sin (\omega t)\right]=\sum_{n=-\infty}^{+\infty} J_{n}\left(\omega_{1} / \omega\right) e^{i n \omega t}$, which is a signature of most photoassisted processes, the tunnel amplitude is cast into an infinite sum:

$$
A(t)=\Gamma_{0} \sum_{n=-\infty}^{+\infty} e^{i\left(\omega_{0}+n \omega\right) t} J_{n}\left(\frac{\omega_{1}}{\omega}\right)
$$

which gives:

$$
\begin{aligned}
& \left\langle I_{B}(t)\right\rangle=\frac{e^{*} i \Gamma_{0}^{2}}{4 \pi^{2} a^{2}} \sum_{n=-\infty}^{+\infty} \sum_{m=-\infty}^{+\infty} J_{n}\left(\frac{\omega_{1}}{\omega}\right) J_{m}\left(\frac{\omega_{1}}{\omega}\right) \sum_{\eta \eta_{1}} \eta_{1} \\
& \times\left(\cos ((n-m) \omega t) \int_{-\infty}^{+\infty} d \tau e^{2 \nu G^{\eta \eta_{1}}(\tau)} \sin \left(\left(\omega_{0}+m \omega\right) \tau\right)+\sin ((n-m) \omega t) \int_{-\infty}^{+\infty} d \tau e^{2 \nu G^{\eta \eta_{1}}(\tau)} \cos \left(\left(\omega_{0}+m \omega\right) \tau\right)\right) .
\end{aligned}
$$


The change of variables $\tau=t-t_{1}$ with $d \tau=-d t_{1}$ has been operated. Note that only $\eta_{1}=-\eta$ terms in Eq. (11) contribute to the first integral because of the symmetry properties of the Green's function. On the contrary, only $\eta_{1}=\eta$ terms contribute to the second integral because such an integral does not depend on $\eta$. Using the expression for the Green's function Eq.(8), the current can thus be split into two contributions, with integrants containing either a sine or a cosine of $\omega_{0}+m \omega$. The time integral can be performed analytically and is expressed in terms of the Gamma function $\Gamma$ (see Appendix A).

Grouping the two contributions, we obtain:

$$
\begin{aligned}
\left\langle I_{B}(t)\right\rangle= & \frac{e^{*} \Gamma_{0}^{2}}{2 \pi a^{2} \Gamma(2 \nu)}\left(\frac{a}{v_{F}}\right)^{2 \nu} \sum_{n=-\infty}^{+\infty} \sum_{m=-\infty}^{+\infty} J_{n}\left(\frac{\omega_{1}}{\omega}\right) J_{m}\left(\frac{\omega_{1}}{\omega}\right) \\
& \times\left(\cos ((n-m) \omega t) \operatorname{sgn}\left(\omega_{0}+m \omega\right)+\sin ((n-m) \omega t) \tan (\pi \nu)\right)\left|\omega_{0}+m \omega\right|^{2 \nu-1} .
\end{aligned}
$$

The Fourier transform of the current, $\left\langle I_{B}(\Omega)\right\rangle=\int e^{i \Omega t}\left\langle I_{B}(t)\right\rangle d t$, yields:

$$
\begin{aligned}
\left\langle I_{B}(\Omega)\right\rangle= & \frac{e^{*} \Gamma_{0}^{2}}{4 \pi a^{2} \Gamma(2 \nu)}\left(\frac{a}{v_{F}}\right)^{2 \nu} \sum_{n=-\infty}^{+\infty} \sum_{m=-\infty}^{+\infty} J_{n}\left(\frac{\omega_{1}}{\omega}\right) J_{m}\left(\frac{\omega_{1}}{\omega}\right) \\
& \times\left(\operatorname{sgn}\left(\omega_{0}+m \omega\right)\left|\omega_{0}+m \omega\right|^{2 \nu-1}(\delta(\Omega+(n-m) \omega)+\delta(\Omega-(n-m) \omega)\right. \\
& +i \tan (\pi \nu)\left|\omega_{0}+m \omega\right|^{2 \nu-1}(\delta(\Omega+(n-m) \omega)-\delta(\Omega-(n-m) \omega)) .
\end{aligned}
$$

To zero order in the amplitude $\omega_{1}$ of the modulation, we recover the I-V characteristics of the pure stationary regime $(n=m=0)$ :

$$
\left\langle I_{B}\right\rangle^{(0)}=\frac{e^{*} \Gamma_{0}^{2}}{2 \pi a^{2} \boldsymbol{\Gamma}(2 \nu)}\left(\frac{a}{v_{F}}\right)^{2 \nu} \operatorname{sgn}\left(\omega_{0}\right)\left|\omega_{0}\right|^{2 \nu-1} .
$$

Expanding the current to first order with $\omega_{1}$ corresponds to $n= \pm 1$ and $m=0$ or to $n=0$ and $m= \pm 1$ :

$$
\begin{aligned}
\left\langle I_{B}(t)\right\rangle^{(1)}= & \frac{e^{*} \Gamma_{0}^{2}}{4 \pi a^{2} \Gamma(2 \nu)}\left(\frac{a}{v_{F}}\right)^{2 \nu} \frac{\omega_{1}}{\omega}\left[2 \sin (\omega t) \tan (\pi \nu)\left|\omega_{0}\right|^{2 \nu-1}\right. \\
& +\cos (\omega t)\left(\operatorname{sgn}\left(\omega_{0}+\omega\right)\left|\omega_{0}+\omega\right|^{2 \nu-1}-\operatorname{sgn}\left(\omega_{0}-\omega\right)\left|\omega_{0}-\omega\right|^{2 \nu-1}\right) \\
& \left.-\sin (\omega t) \tan (\pi \nu)\left(\left|\omega_{0}+\omega\right|^{2 \nu-1}+\left|\omega_{0}-\omega\right|^{2 \nu-1}\right)\right] .
\end{aligned}
$$

In the limit $\nu=1$, Eqs. (14) and (15) lead, at zero and first orders with $\omega_{1}$, to the backscattering current :

$$
\left\langle I_{B}(t)\right\rangle=\frac{e^{*} \Gamma_{0}^{2}}{4 \pi v_{F}^{2}}\left(\omega_{0}+\omega_{1} \cos (\omega t)\right) .
$$

Finally, one can analyze which DC contribution is provided by the AC modulation: the rectification property. This information is contained in the zero-frequency Fourier transform:

$$
\begin{aligned}
\left\langle I_{B}(\Omega=0)\right\rangle= & \frac{e^{*} \Gamma_{0}^{2}}{2 \pi a^{2} \Gamma(2 \nu)}\left(\frac{a}{v_{F}}\right)^{2 \nu} \sum_{n=-\infty}^{+\infty} J_{n}^{2}\left(\frac{\omega_{1}}{\omega}\right) \\
& \times \operatorname{sgn}\left(\omega_{0}+n \omega\right)\left|\omega_{0}+n \omega\right|^{2 \nu-1},
\end{aligned}
$$

which is obtained to all orders in the modulation. Also note that it can be re-expressed in terms of the DC current:

$$
\left\langle I_{B}(\Omega=0)\right\rangle=\sum_{n=-\infty}^{+\infty} J_{n}^{2}\left(\frac{\omega_{1}}{\omega}\right)\left\langle I_{B}\right\rangle_{\omega_{0} \rightarrow \omega_{0}+n \omega}^{(0)},
$$

a general relation which was noticed in Ref. 31 .

\section{B. Validity condition}

First, note that the result of Eq. (12) seems to blow up at $\nu=1 / 2$. In this situation, the absolute value in the last term of this equation becomes independent of $m$ and the term proportional to $\tan (\pi \nu)$ vanishes at $\nu=1 / 2$. 
Consider now the case of arbitrary $\nu$. For the present result to be valid, one should be consistent with the assumption of weak backscattering, and the differential conductance should thus be much smaller than the conductance quantum $\nu e^{2} / \hbar$ associated with the unperturbed fractional edge:

$$
\frac{\partial\left\langle I_{B}(t)\right\rangle}{\partial \omega_{0}} \ll \frac{e}{2 \pi} .
$$

First, consider the limit of a weak AC perturbation $\omega_{1} / \omega \ll 1$. In this case, the zero order current given by Eq. (14) dominates and,

$$
\frac{\partial\left\langle I_{B}\right\rangle^{(0)}}{\partial \omega_{0}}=\frac{e \nu(2 \nu-1) \Gamma_{0}^{2}}{2 \pi a^{2} \boldsymbol{\Gamma}(2 \nu)}\left(\frac{a}{v_{F}}\right)^{2 \nu}\left|\omega_{0}\right|^{2 \nu-2}
$$

In particular, for $\nu=1$, the condition of validity of perturbation theory yields:

$$
\Gamma_{0}^{2} \ll v_{F}^{2},
$$

which is independent of $\omega_{0}$, and which simply states that electron transport along the edge dominates over backscattering contributions.

For Laughlin fractions which have $\nu<1$, the backscattering current diverges at low bias (the known "paradox" of Luttinger liquids), and the validity condition for a weak AC modulation reads:

$$
\left|\omega_{0}\right| \gg\left|\frac{a^{2} \boldsymbol{\Gamma}(2 \nu)}{\nu(2 \nu-1) \Gamma_{0}^{2}}\left(\frac{v_{F}}{a}\right)^{2 \nu}\right|^{(2 \nu-2)^{-1}} .
$$

One can also derive a validity condition if the limit where $\omega_{1} / \omega$ is arbitrary. The starting point is to rewrite Eq. (12) by resumming over the integer variable $n$ :

$$
\begin{aligned}
\left\langle I_{B}(t)\right\rangle= & \frac{e^{*} \Gamma_{0}^{2}}{2 \pi a^{2} \boldsymbol{\Gamma}(2 \nu)}\left(\frac{a}{v_{F}}\right)^{2 \nu} \sum_{m=-\infty}^{+\infty} J_{m}\left(\frac{\omega_{1}}{\omega}\right) \\
& \times\left(\cos \left(\frac{\omega_{1}}{\omega} \sin (\omega t)-m \omega t\right) \operatorname{sgn}\left(\omega_{0}+m \omega\right)+\sin \left(\frac{\omega_{1}}{\omega} \sin (\omega t)-m \omega t\right) \tan (\pi \nu)\right)\left|\omega_{0}+m \omega\right|^{2 \nu-1} .
\end{aligned}
$$

Every time $\omega_{0}$ approaches $m \omega$, the backscattering current $\left\langle I_{B}(t)\right\rangle$ diverges because of the quasiparticle density of states exponent $\left|\omega_{0}-m \omega\right|^{2 \nu-1}$. If one treats all these divergences (for different $m$ ) independently, one can derive a sufficient condition for the validity of Eq. (12):

$$
\left|\omega_{0}-m \omega\right| \gg\left|\frac{a^{2} \boldsymbol{\Gamma}(2 \nu)}{\nu(2 \nu-1) \Gamma_{0}^{2}}\left(\frac{v_{F}}{a}\right)^{2 \nu}\right|^{(2 \nu-2)^{-1}} .
$$

Note that this condition looks quite similar to the one derived for the weak AC modulation, except for the shift mw on the voltage bias.

In the following section, a series of plots (Figs. 25) displays the zero frequency component of the current, the noise and the noise derivative with respect to bias all as a function of the ratio $\omega_{0} / \omega$, for various filling factors. The divergences on these plots should be understood to be unphysical outside the limits specified by Eq. (24). Outside these limits, one expects a crossover to the strong backscattering regime, in analogy with the renormalization group analysis of Ref. 32 for the stationary situation.

\section{Photo-assisted shot noise}

The symmetrized backscattering current noise correlator is expressed with the help of the Keldysh contour:

$$
\begin{aligned}
S\left(t, t^{\prime}\right) & =\frac{1}{2}\left\langle I_{B}(t) I_{B}\left(t^{\prime}\right)\right\rangle+\frac{1}{2}\left\langle I_{B}\left(t^{\prime}\right) I_{B}(t)\right\rangle-\left\langle I_{B}(t)\right\rangle\left\langle I_{B}\left(t^{\prime}\right)\right\rangle \\
& =\frac{1}{2} \sum_{\eta}\left\langle T_{K}\left\{I_{B}\left(t^{\eta}\right) I_{B}\left(t^{\prime-\eta}\right) e^{-i \int_{K} d t_{1} H_{B}\left(t_{1}\right)}\right\}\right\rangle .
\end{aligned}
$$


Here one is interested in the Poissonian limit only, so in the weak backscattering case, one collects the second order contribution in the tunnel barrier amplitude $A(t)$, and the product of the average backscattering current can be dropped. The meaning of the Poissonian limit is that quasiparticles which tunnel from one edge to another do so in an independent manner. Yet by doing so they can absorb or emit $m$ "photon" quanta of $\omega$ ( $m$ integer).

Eq. (25) is our definition of the real time correlator, but this choice is not necessarily obvious. Indeed, it has been pointed out in Ref. 36 that the choice of correlator - symmetrized or non symmetrized - depends on how the noise measurement is performed. More recently, Ref. 37 has argued that the non-symmetrized correlator corresponds to what is actually probed at finite frequencies, provided that the detector which is used to measure the noise is set at sufficiently low temperatures. In the DC regime however, it is well understood that such differences do not matter, as long as one considers zero frequency noise. In the present work, the origin for off-equilibrium phenomena comes from both a DC bias and an AC drive. The latter effect breaks time translational invariance, so that the finite frequency noise involves in general two frequencies $\frac{16}{}$ :

$$
S\left(\Omega_{1}, \Omega_{2}\right)=\iint e^{i\left(\Omega_{1} t+\Omega_{2} t^{\prime}\right)} S\left(t, t^{\prime}\right) d t d t^{\prime} .
$$

Here, one of the main purpose of this work is to analyze the noise when both frequencies $\Omega_{1}$ and $\Omega_{2}$ are set to zero, because the presence of the $\mathrm{AC}$ perturbation mimics at finite frequency noise measurement. For zero frequencies, it is therefore justified to use the symmetrized correlator of Eq. (25).

The quasiparticle correlators have already been calculated for the backscattering current, therefore in terms of chiral Green's functions the real time correlator becomes:

$$
S\left(t, t^{\prime}\right)=\frac{\left(e^{*}\right)^{2}}{8 \pi^{2} a^{2}} \sum_{\eta} e^{2 \nu G^{\eta-\eta}\left(t, t^{\prime}\right)}\left(A(t) A^{*}\left(t^{\prime}\right)+A^{*}(t) A\left(t^{\prime}\right)\right) .
$$

The double Fourier transform of the real time noise correlator then reads:

$$
\begin{aligned}
S\left(\Omega_{1}, \Omega_{2}\right)= & \frac{\left(e^{*}\right)^{2} \Gamma_{0}^{2}}{4 \pi^{2} a^{2}} \sum_{n=-\infty}^{+\infty} \sum_{m=-\infty}^{+\infty} J_{n}\left(\frac{\omega_{1}}{\omega}\right) J_{m}\left(\frac{\omega_{1}}{\omega}\right) \\
& \times \sum_{\eta} \iint d t d t^{\prime} e^{i\left(\Omega_{1} t+\Omega_{2} t^{\prime}\right)} e^{2 \nu G^{\eta-\eta}\left(t, t^{\prime}\right)} \cos \left(\omega_{0}\left(t-t^{\prime}\right)+\omega\left(n t-m t^{\prime}\right)\right) .
\end{aligned}
$$

Changing to relative time coordinates $\tau=t-t^{\prime}$ and $\tau^{\prime}=t+t^{\prime}$, one obtains two contributions whose time integrals can be factored out, and the time integrals can be performed (see Appendix $\underline{\mathrm{A}}$ ):

$$
\begin{aligned}
S\left(\Omega_{1}, \Omega_{2}\right) & =\frac{\left(e^{*}\right)^{2} \Gamma_{0}^{2}}{4 \pi a^{2} \boldsymbol{\Gamma}(2 \nu)}\left(\frac{a}{v_{F}}\right)^{2 \nu} \sum_{n=-\infty}^{+\infty} \sum_{m=-\infty}^{+\infty} J_{n}\left(\frac{\omega_{1}}{\omega}\right) J_{m}\left(\frac{\omega_{1}}{\omega}\right) \\
& \times\left(\left|\Omega_{1}+\omega_{0}+n \omega\right|^{2 \nu-1} \delta\left(\Omega_{1}+\Omega_{2}+(n-m) \omega\right)+\left|\Omega_{1}-\omega_{0}-n \omega\right|^{2 \nu-1} \delta\left(\Omega_{1}+\Omega_{2}-(n-m) \omega\right)\right) .
\end{aligned}
$$

In particular, the case where both frequencies are set to zero can serve as a point of comparison with experiments:

$$
S(0,0)=\frac{\left(e^{*}\right)^{2} \Gamma_{0}^{2}}{2 \pi a^{2} \boldsymbol{\Gamma}(2 \nu)}\left(\frac{a}{v_{F}}\right)^{2 \nu} \sum_{n=-\infty}^{+\infty} J_{n}^{2}\left(\frac{\omega_{1}}{\omega}\right)\left|\omega_{0}+n \omega\right|^{2 \nu-1} .
$$

Note that this quantity is obtained in perturbation theory but it contains all orders in the harmonic perturbation.

The results are now illustrated and discussed. The quantities which are plotted on Figs. 2 to 6 are normalized according to the prefactors which appear on Eqs. (17) and (30). At $\nu=1$, one recovers (Fig. 21) the results of Ref. 15. The noise derivative with respect to $\omega_{0}$ has a step-like behavior, with steps located at $\omega_{0} / \omega= \pm n,(n$ integer $)$, and the step heights are related to the weight $J_{n}^{2}\left(\omega_{1} / \omega\right)$. The zero frequency Fourier transform of the current does not display any structure at the step locations for the noise: it is simply linear. For lower values of the filling factor, electronic correlations modify this behavior drastically. Generally speaking, the power law behavior $\left|\omega_{0}+n \omega\right|^{2 \nu-1}$ should have a "stronger" singularity for lower filling factors. For the fractional quantum Hall effect, only odd fractions are allowed for $\nu$. The results are illustrated with filling factor $\nu=1 / 3$, which represents the correlated state which is "easiest" to access experimentally 10.11 . First, one observes that contrary to the free electron case, the zero frequency Fourier transform of the current displays singularities every time $\omega_{0} / \omega$ reaches an integer value (Fig. 3). Such singularities 


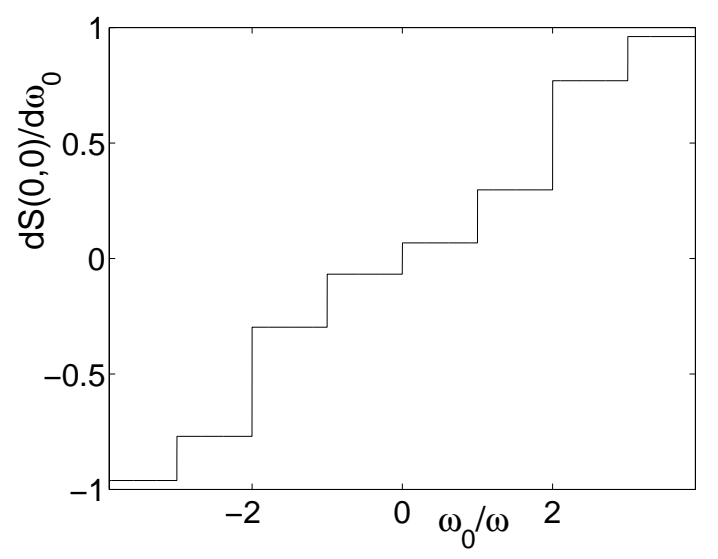

FIG. 2: Derivative of the zero frequency backscattering noise (arbitrary units) as a function of bias voltage, for $\nu=1$, for a drive amplitude $\omega_{1} / \omega=3$.

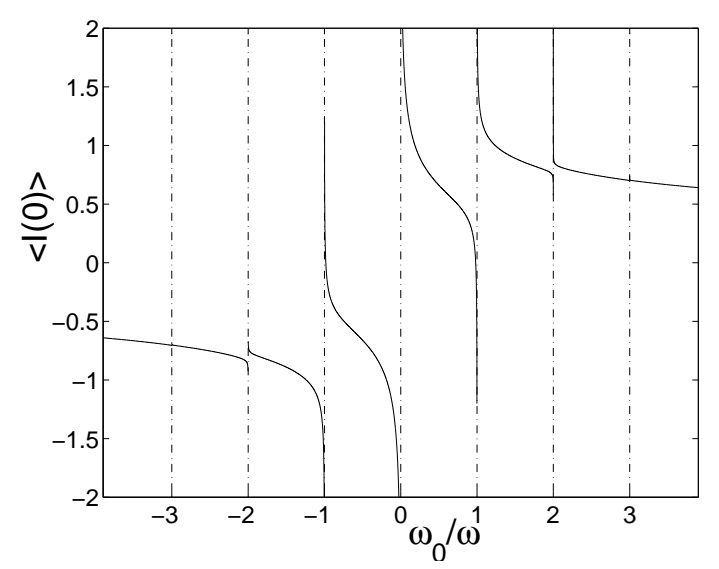

FIG. 3: Zero frequency Fourier transform of the backscattering current, as a function of bias voltage, for $\nu=1 / 3, \omega_{1} / \omega=1$.

reflect the tunneling density of states of the quasiparticles, in a similar manner which is observed in the backscattering current-voltage characteristics ${ }^{32}$. Nevertheless, one should remember that the present calculation is only valid in the weak backscattering regime: the current $\langle I(t)\rangle$ whose Fourier transform is displayed in Fig. 3] should be much lower than the maximal current along the fractional edge as it is discussed in section IIIB

Next, the noise (for both frequencies set to zero) is plotted as a function of bias. While at $\nu=1$, the noise was found to be a continuous function of $\omega_{0}$ (with discontinuous derivatives), for $\nu=1 / 3$ it displays evenly spaced singularities as for the current (Fig. 4). Note that the spacing between singular points can serve as a diagnosis for the effective charges at play, as in the DC shot noise experiments: recall that the effective charge $e^{*}=\nu e$ is implicit in the definition of $\omega_{0}$. Measured at a given, fixed amplitude for the noise, the peaks widths get reduced as $\left|\omega_{0} / \omega\right|$ is increased: on the figure it becomes difficult to resolve this width beyond $\left|\omega_{0} / \omega\right| \sim 3$.

The present results bear strong similarities with the finite frequency noise of Ref. 8 (Eq. (17)), which was computed in the absence of an AC bias. In fact Eq. (29) contains these previous results. In the absence of the AC bias $\left(\omega_{1} \rightarrow 0\right)$, only $n=m=0$ remains in Eq. (29), giving a dependence $\delta\left(\Omega_{1}-\Omega_{2}\right)$. Using the definition of the double Fourier transform, one identifies $\Omega_{1}$ as the frequency where the noise is computed in Ref. [8. Indeed, in the absence of an $\mathrm{AC}$ bias, time translational invariance is restored and this frequency is the only relevant one, and one gets the same density of states divergences as for the DC, finite frequency noise. The interesting feature in the present work is that when one considers the AC driven noise, with both frequencies set to zero, one recovers information on a DC, finite frequency measurement.

Another point of comparison is the (unphysical) value $\nu=1 / 2$ : although this filling factor does not correspond to a Laughlin fraction, it allows to make a connection with an exact solution using the refermionization procedure ${ }^{8.32}$ (Sec. 5). The backscattering current noise shows no structure: this noise is perfectly flat with respect to the bias voltage because of the tunneling density of states exponents. On the other hand, the zero-frequency current displays steps as 


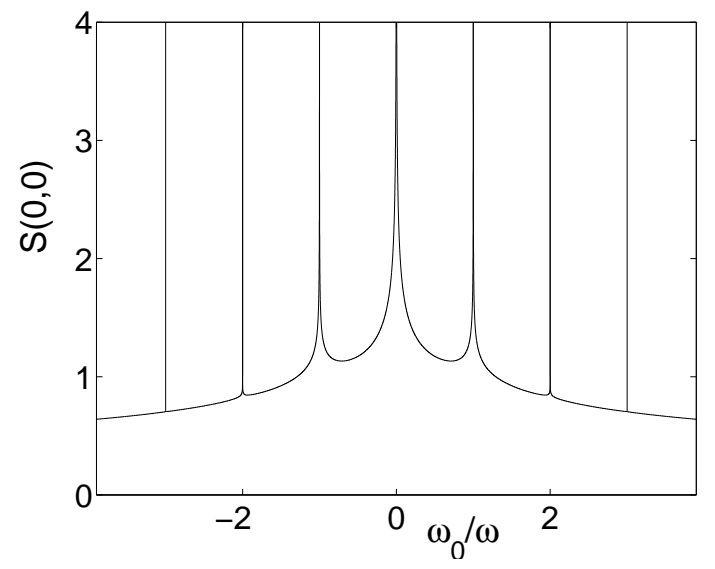

FIG. 4: Zero frequency backscattering noise as a function of bias voltage, for $\nu=1 / 3, \omega_{1} / \omega=1$.

a function of $\omega_{0} / \omega$ (Fig. 5). Here, one can make a direct comparison between the zero frequency noise derivative at $\nu=1$ and the zero Fourier transform of the current at $\nu=1 / 2$ : the frequency exponents for both quantities turn out to be the same, and the Bessel coefficients which appear in front of these terms are also identical, in Eqs. (17) and (30).

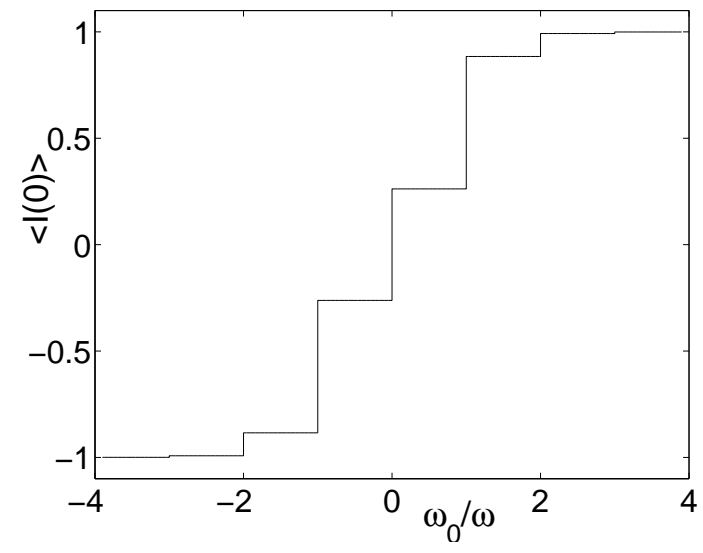

FIG. 5: Zero frequency backscattering current as a function of bias voltage, for $\nu=1 / 2, \omega_{1} / \omega=3 / 2$.

\section{STRONG BACKSCATTERING LIMIT}

The results of the preceding section can be straightforwardly extended to treat the limit of strong backscattering, by appealing to the duality relation between the weak and strong backscattering limits. Indeed, the action of a gate voltage separates the fractional quantum Hall fluid into two components, between which only electrons can tunnel. The main changes to operate on the preceding results are in the tunneling operator, as the fields now represent normal electrons. This corresponds to the substitution:

$$
\begin{aligned}
\nu & \rightarrow \frac{1}{\nu}, \\
e * & \rightarrow e .
\end{aligned}
$$

This yields for the electron tunneling current and noise:

$$
\begin{aligned}
\langle I(t)\rangle= & \frac{e \Gamma_{0}^{2}}{2 \pi a^{2} \boldsymbol{\Gamma}(2 / \nu)}\left(\frac{a}{v_{F}}\right)^{2 / \nu} \sum_{n=-\infty}^{+\infty} \sum_{m=-\infty}^{+\infty} J_{n}\left(\frac{\omega_{1}}{\omega}\right) J_{m}\left(\frac{\omega_{1}}{\omega}\right) \\
& \times\left(\cos ((n-m) \omega t) \operatorname{sgn}\left(\omega_{0}+m \omega\right)+\sin ((n-m) \omega t) \tan (\pi / \nu)\right)\left|\omega_{0}+m \omega\right|^{2 / \nu-1},
\end{aligned}
$$




$$
\begin{aligned}
S\left(\Omega_{1}, \Omega_{2}\right) & =\frac{e^{2} \Gamma_{0}^{2}}{4 \pi a^{2} \boldsymbol{\Gamma}(2 / \nu)}\left(\frac{a}{v_{F}}\right)^{2 / \nu} \sum_{n=-\infty}^{+\infty} \sum_{m=-\infty}^{+\infty} J_{n}\left(\frac{\omega_{1}}{\omega}\right) J_{m}\left(\frac{\omega_{1}}{\omega}\right) \\
& \times\left(\left|\Omega_{1}+\omega_{0}+n \omega\right|^{2 / \nu-1} \delta\left(\Omega_{1}+\Omega_{2}+(n-m) \omega\right)+\left|\Omega_{1}-\omega_{0}-n \omega\right|^{2 / \nu-1} \delta\left(\Omega_{1}+\Omega_{2}-(n-m) \omega\right)\right) .
\end{aligned}
$$

In particular, for zero frequencies,

$$
S(0,0)=\frac{e^{2} \Gamma_{0}^{2}}{2 \pi a^{2} \boldsymbol{\Gamma}(2 / \nu)}\left(\frac{a}{v_{F}}\right)^{2 / \nu} \sum_{n=-\infty}^{+\infty} J_{n}^{2}\left(\frac{\omega_{1}}{\omega}\right)\left|\omega_{0}+n \omega\right|^{2 / \nu-1} .
$$

The results are displayed in Fig. 6] On the one hand, one can expect that the photo-assisted shot noise should

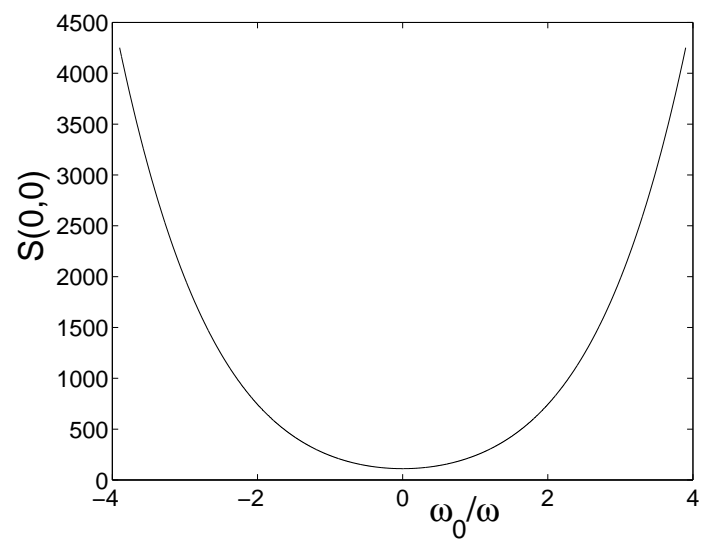

FIG. 6: Zero frequency noise as a function of bias voltage, for $\nu=1 / 3, \omega_{1} / \omega=3$.

bear similarities with the free electron case because the charge carriers which tunnel from one quantum Hall fluid to the other are electrons. On the other hand, the transport occurs between two correlated electron systems with a vanishing tunneling density of states. Here, we see that the photo-assisted shot noise is a smooth function of $\omega_{0} / \omega$. This suggests that the strong backscattering regime does not offer any straightforward diagnosis for effective charges as in the weak backscattering case.

Finally, for the sake of completeness, we mention that for fractional quantum Hall systems in the strong backscattering regime, super-Poissonian noise has been observed in DC shot noise measurements. Indeed, it has been shown that $\mathrm{t}^{38}$ at sufficiently low temperatures, the effective charge which tunnels from one quantum Hall fluid to the other, as analyzed by the ratio $S(\omega=0) /\langle I(t)\rangle$, can be equal to $2 e$ or can be even larger. These experiments suggest that the relevant tunneling process involves pairs - or groups - of electrons. From the point of view of our model, this means that one should take into account the tunneling of such pairs, while an inspection of the renormalization group equations tells us that such processes should be less relevant than the bare electron tunneling term. While this topic is still under debate, and has no simple theoretical explanation for the DC shot noise, here can only give a schematic picture of what will happen for the photo-assisted shot noise. In the above, one recalls that the vanishing of the density of states for single electron tunneling in a Luttinger liquid is the reason why photo-assisted shot noise does not display any sharp features for the strong backscattering regime. The density of states for two electron tunneling is expected to vanish with an exponent $4 / \nu-1$, i.e. much faster than the single electron case. We thus expect no sharp features to show up in the photo-assisted shot noise at integer values of $\omega_{0} / \omega$.

\section{EXACT SOLUTION AT $\nu=1 / 2$}

\section{A. Refermionization in the presence of an AC perturbation}

In order to gain further insights for the backscattering current away from the tunneling regime, we study the limit $\nu=1 / 2$ where it is possible to refermionize. The various chiral current correlators which appear in the calculation of 
the DC backscattering noise and in the DC shot noise have been computed in this manner in Ref. 8. The refermionized model involves the combinations of edge state densities:

$$
\rho_{ \pm}(x) \equiv \rho_{R}(x) \pm \rho_{L}(-x) .
$$

$\rho_{+}$describes a free boson theory, whereas boundary and scattering effects are included in the solution for the fermion field operator $\psi$ which specifies $\rho_{-}=\psi^{\dagger} \psi$. The Hamiltonian which specifies the dynamics of this latter field is:

$$
H_{-}=\int d x\left\{\psi^{\dagger}(x)\left[-i \partial_{x}-\omega_{0}-\omega_{1} \cos (\omega t)\right] \psi(x)+\sqrt{2 \pi} \delta(x)\left[\Gamma_{0} \psi^{\dagger}(x) f+\Gamma_{0}^{*} f \psi(x)\right]\right\},
$$

were $f$ is a Majorana Fermion which satisfies $\{f, f\}=2$ and commutes with $\psi$. Note that the total bias voltage appears explicitly in the Hamiltonian because a gauge transformation has been used on $\psi$. In the presence of an $\mathrm{AC}$ voltage modulation, the Heisenberg equations of motion become:

$$
\begin{aligned}
-i \partial_{t} \psi(x, t) & =\left(i \partial_{x}+\omega_{0}+\omega_{1} \cos (\omega t)\right) \psi(x, t)+\sqrt{2 \pi} \Gamma_{0} f(t) \delta(x), \\
-i \partial_{t} \psi^{\dagger}(x, t) & =\left(i \partial_{x}-\omega_{0}-\omega_{1} \cos (\omega t)\right) \psi^{\dagger}(x, t)-\sqrt{2 \pi} \Gamma_{0} f(t) \delta(x), \\
-i \partial_{t} f(t) & =2 \sqrt{2 \pi} \Gamma_{0}\left(\psi(0, t)-\psi^{\dagger}(0, t)\right) .
\end{aligned}
$$

The general solutions allow for a mismatch of the field operators at the impurity location:

$$
\begin{aligned}
\psi(x, t) & =\sum_{\Omega}\left(A_{\Omega} \Theta(-x)+B_{\Omega} \Theta(x)\right) e^{i\left(\Omega+\omega_{0}\right) x-i \Omega t+i \frac{\omega_{1}}{\omega} \sin (\omega t)}, \\
\psi^{\dagger}(x, t) & =\sum_{\Omega}\left(A_{-\Omega}^{\dagger} \Theta(-x)+B_{-\Omega}^{\dagger} \Theta(x)\right) e^{i\left(\Omega-\omega_{0}\right) x-i \Omega t-i \frac{\omega_{1}}{\omega} \sin (\omega t)} .
\end{aligned}
$$

The matching condition for the $A_{\Omega}$ and $B_{\Omega}$ are obtained by integrating the equations of motion over the singularity:

$$
i \partial_{t}\left[\psi\left(0_{+}, t\right)-\psi\left(0_{-}, t\right)\right]+2 i \pi \Gamma_{0}^{2}\left[\psi\left(0_{+}, t\right)+\psi\left(0_{-}, t\right)-\psi^{\dagger}\left(0_{+}, t\right)-\psi^{\dagger}\left(0_{-}, t\right)\right]=0 .
$$

This condition does not contain the DC voltage, nor the time dependent drive. One then substitutes the general solution for $\psi\left(0_{ \pm}\right)$in the latter equation, and subsequently, one uses the generating function of the Bessel function to obtain a the general relationship between the Fourier components $A_{\Omega \pm n \omega}$ 's and $B_{\Omega \pm n \omega}$ 's. The details of this calculation are explicited in Appendix B] Note that unlike Ref. 30, the presence of the harmonic perturbation couples the Fourier components at different frequencies. Because here, we are interested in obtaining the modulation of the backscattering current as a first step, it is sufficient to solve such equation to $O\left(\omega_{1}\right) . B_{\omega}$ and $B_{-\omega}^{\dagger}$ are given by Eqs. (B7) and (B8). This completes the refermionization procedure in the presence of the harmonic time perturbation.

Note that a previous work on the photo-assisted current in the same geometry ${ }^{31}$ claims to have a general result for this situation, arguing that the AC modulation can be gauged-out by the transformation: $\psi(x, t)=$ $e^{i \omega_{1} \sin (\omega t) / 2 \omega} \tilde{\psi}(x, t)$. Here, we note that the matching conditions used for this new field is:

$$
\left(i \partial_{t}+\omega_{1} \cos (\omega t) / 2\right)\left[\tilde{\psi}\left(0_{+}, t\right)-\tilde{\psi}\left(0_{-}, t\right)\right]+2 i \pi \Gamma_{0}^{2}\left[\tilde{\psi}\left(0_{+}, t\right)+\tilde{\psi}\left(0_{-}, t\right)-\tilde{\psi}^{\dagger}\left(0_{+}, t\right)-\tilde{\psi}^{\dagger}\left(0_{-}, t\right)\right]=0,
$$

so the matching condition itself becomes time dependent with this choice of gauge, because of the harmonic perturbation. This time contribution was discarded in Ref. 31 .

\section{B. Backscattering current}

The average backscattering current can be expressed in terms of the fermion density as follows:

$$
\left\langle I_{B}(t)\right\rangle=\frac{1}{2}\left\langle\rho_{-}\left(x_{-}, t\right)-\rho_{-}\left(x_{+}, t\right)\right\rangle .
$$

The details of the calculation are explained in Appendix B The general result for the backscattering current reads:

$$
\begin{aligned}
\left\langle I_{B}(t)\right\rangle= & 4 \pi \Gamma_{0}^{2} \tan ^{-1}\left(\frac{\omega_{0}}{4 \pi \Gamma_{0}^{2}}\right)+4 \pi \Gamma_{0}^{2} \frac{\omega_{1}}{2 \omega} \cos (\omega t)\left(\tan ^{-1}\left(\frac{\omega_{0}+\omega}{4 \pi \Gamma_{0}^{2}}\right)-\tan ^{-1}\left(\frac{\omega_{0}-\omega}{4 \pi \Gamma_{0}^{2}}\right)\right) \\
& -4 \pi \Gamma_{0}^{2} \frac{\omega_{1}}{2 \omega} \sin (\omega t)\left(\ln \left(\omega_{0}^{2}+16 \pi^{2} \Gamma_{0}^{4}\right)-\frac{1}{2} \ln \left(\left(\omega_{0}+\omega\right)^{2}+16 \pi^{2} \Gamma_{0}^{4}\right)-\frac{1}{2} \ln \left(\left(\omega_{0}-\omega\right)^{2}+16 \pi^{2} \Gamma_{0}^{4}\right)\right),
\end{aligned}
$$


which is the final result for the backscattering current computed using the refermionization procedure, to first order in the harmonic perturbation. The perturbative result of Eq. (15) can be recovered by choosing the limit $\Gamma_{0}^{2} \ll\left|\omega_{0}\right|$, $|\omega| \ll\left|\omega_{0}\right|:$

$$
\left\langle I_{B}(t)\right\rangle=2 \pi^{2} \Gamma_{0}^{2} \operatorname{sgn}\left(\omega_{0}\right)+2 \pi^{2} \Gamma_{0}^{2} \frac{\omega_{1}}{2 \omega} \cos (\omega t)\left(\operatorname{sgn}\left(\omega_{0}+\omega\right)-\operatorname{sgn}\left(\omega_{0}-\omega\right)\right) .
$$

We thus recover the perturbative result of Eqs. (14) and (15) when $\nu=1 / 2$.

\section{SUMMARY AND CONCLUSION}

Photo-assisted shot noise makes it possible to probe the transport properties of mesoscopic conductors in the time domain, as it is provides information which is directly related to the spectral density of noise. So far, such information has mainly focused on mesoscopic conductors where electron-electron interactions do not intervene, except via screening effects in the RPA approximation 23 . The present study deals with the possible diagnosis of the "simplest" one dimensional correlated electron systems - a fractional quantum Hall bar with two counter-propagating edge excitations - and showed in particular that the singularities in the noise and current provide information on the effective charges corresponding to Laughlin fractions for the quantum Hall fluid. Although the present work bears close ties with previous work on finite frequency noise $\mathrm{e}^{30}$ in the fractional quantum Hall effect, a first principle derivation of photo-assisted shot noise in these systems was still lacking.

The bulk of the present results were derived in the context of perturbation theory, using two opposite limits (weak and strong backscattering) and shows that a direct diagnosis of quasiparticle tunneling can be reached for weak backscattering situations, while in the limit of strong backscattering, one does not expect much of a structure in the photo-assisted shot noise. For a correlated electron state such as $\nu=1 / 3$, the noise and backscattering current for a weak impurity contain singularities at integer values of the fraction $\omega_{0} / \omega$. At finite temperatures however, these singularities will be smeared out and the peak heights should scale as $\left(k_{B} T\right)^{-1}$ as in Ref. 16, while they will also acquire thermal broadening. The condition for resolving the peaks in then $k_{B} T<\omega_{0}$, similarly to the temperature crossover of the DC regime. If one is to compare this diagnosis with the measurement of the Fano factor in a DC shot noise measurement with the present procedure, one can argue that the extra degree of freedom provided by the probe frequency $\omega$ facilitates the monitoring of such singularities. A similar structure does not show up in the strong backscattering regime because, in this case, electron tunneling density of states are involved, rather than quasiparticle tunneling density of states. This suggest that photo-assisted transport could be used to probe, in future experiments, the crossover from the strong to the weak backscattering regimes in the fractional quantum Hall effect.

In order to go beyond perturbation theory, a systematic, perturbative approach in the drive amplitude, was proposed, based on the refermionization procedure for $\nu=1 / 2$. The photo-assisted current was derived to first order in this amplitude in the present work, but generalizations of this method, concerning the study of the rectification property of the current (its zero frequency Fourier transform) or the photo-assisted shot noise are possible in principle, but they remain a challenge because of the need to pursue the calculation to higher orders in the drive amplitude.

Note that the present work treats both edges as independent entities, which are only coupled via the tunneling Hamiltonian of Eq. (2). A more precise model would include density-density interactions between both edges in the vicinity of the impurity, for instance described by a screened Coulomb interaction. Such interactions were considered for electron wave guides containing two coupled non-chiral Luttinger liquids in section 7 of Ref. 39, and the transport properties of this system through an impurity were analyzed in Ref. 40, assuming that the interaction is effective over the whole length of the wire. Here however, one focuses on a situation where the edge excitations have a chiral character. For the integer quantum Hall effect, the screened interaction between two electron edge states were discussed previously ${ }^{41}$. This interaction alone - over a finite length which characterizes the regions where the two edges are in close proximity - is understood to lead to backscattering of the collective excitations. Indeed, the interaction between edges will give rise to a local inhomogeneity of the Luttinger liquid interaction parameter, as seen in Ref. 42. Although a full generalization to the fractional quantum Hall situation is not fully available, it plausible that the same applies to the present situation. In the presence of such screened interaction, in the vicinity of the impurity, the "proper" excitations would mix right and left moving excitations, so that the tunneling Hamiltonian and current operator would have to be rewritten in terms of new excitations which diagonalize the Hamiltonian. One then expects for backscattering to be enhanced by the screened Coulomb interaction. Nevertheless, such inter-edge interaction will have a lesser role if the tunneling between the two edge states is weak, which is a working assumption of the present work. In fact, such considerations should first be addressed for a DC shot noise measurement in the FQHE alone, rather than in the present work, whose goal is to focus mainly on photo-assisted transport.

Several perspectives can be foreseen in the future. Here, only simple Laughlin fractions have been considered. Yet the present approach - probing transport in a correlated electron system with an external parameter (the drive 
frequency) - in more complicated fractions of the quantum Hall effect is highly relevant, because the charges at play depend on the geometry and the gating applied to the Hall bar ${ }^{43}$. Another possible extension of the present results concerns non-chiral Luttinger liquids, which find experimental applications in semiconductor quantum wires as well as for transport through carbon nanotubes. Overall, photo-assisted transport constitutes a first step for studying the behavior of mesoscopic systems in non-stationary situations, which is relevant here for the understanding of the control of charge injection in the fractional quantum Hall effect.

\section{Acknowledgments}

One of us (T.M.) wishes to thank NTT Basic Research Laboratories for their hospitality. Early discussions with G.B. Lesovik on photo-assisted shot noise are gratefully acknowledged.

\section{APPENDIX A: USEFUL INTEGRALS}

The following integrals are commonly used in the perturbation theory of Luttinger liquids:

$$
\begin{aligned}
\int_{-\infty}^{+\infty} \frac{\sin \left(\omega_{0} \tau\right) d \tau}{\left(\frac{a}{v_{F}}-i \eta \tau\right)^{2 \nu}} & \approx i \pi \eta \operatorname{sgn}\left(\omega_{0}\right) \frac{\left|\omega_{0}\right|^{2 \nu-1}}{\Gamma(2 \nu)} \\
\int_{-\infty}^{+\infty} \frac{\cos \left(\omega_{0} \tau\right) d \tau}{\left(\frac{a}{v_{F}}-i \eta \tau\right)^{2 \nu}} & \approx \pi \frac{\left|\omega_{0}\right|^{2 \nu-1}}{\boldsymbol{\Gamma}(2 \nu)} \\
\int_{-\infty}^{+\infty} d \tau \frac{\cos \left(\left(\omega_{0}+m \omega\right) \tau\right)}{\left(1+i \eta|\tau| v_{F} / a\right)^{2 \nu}} & \approx\left(\frac{a}{v_{F}}\right)^{2 \nu} \frac{\pi\left|\omega_{0}+m \omega\right|^{2 \nu-1} e^{-i \eta \pi \nu}}{\boldsymbol{\Gamma}(2 \nu) \cos (\pi \nu)}
\end{aligned}
$$

with $\eta$ a Keldysh index.

\section{APPENDIX B: $\nu=1 / 2$ SOLUTION}

The system of equations obtained from the general solution for $\psi(x, t)$ and from its matching condition Eq. (43) reads:

$$
\begin{aligned}
\sum_{\Omega}\left[\left(\Omega-\omega_{1} \cos (\omega t)\right)\left(B_{\Omega}-A_{\Omega}\right)+2 i \pi \Gamma_{0}^{2}\left(A_{\Omega}+B_{\Omega}\right)\right] e^{-i \Omega t+i \frac{\omega_{1}}{\omega} \sin (\omega t)} & =\sum_{\Omega} 2 i \pi \Gamma_{0}^{2}\left(A_{-\Omega}^{\dagger}+B_{-\Omega}^{\dagger}\right) e^{-i \Omega t-i \frac{\omega_{1}}{\omega} \sin (\omega t)}, \\
\sum_{\Omega}\left[\left(\Omega+\omega_{1} \cos (\omega t)\right)\left(B_{-\Omega}^{\dagger}-A_{-\Omega}^{\dagger}\right)+2 i \pi \Gamma_{0}^{2}\left(A_{-\Omega}^{\dagger}+B_{-\Omega}^{\dagger}\right)\right] e^{-i \Omega t-i \frac{\omega_{1}}{\omega} \sin (\omega t)} & =\sum_{\Omega} 2 i \pi \Gamma_{0}^{2}\left(A_{\Omega}+B_{\Omega}\right) e^{-i \Omega t+i \frac{\omega_{1}}{\omega} \sin (\omega t)},
\end{aligned}
$$

which corresponds to an infinite system of coupled equations for the Fourier components $A_{\Omega \pm \omega}$ and $B_{\Omega \pm \omega}$. After using the generating function for the Bessel function, one expands each Bessel function in powers of $\omega_{1} / \omega$. This then provides a systematic method of solution, identifying the contribution of each harmonic $\exp [i(\Omega+n \omega) t]$. The zero order solution is the one of Ref. 8. Here we restrict ourselves to first order, which allows to compute the current modulation generated by the perturbation. This corresponds to the set of equations:

$$
\begin{aligned}
& \left(2 i \pi \Gamma_{0}^{2}-\Omega\right)\left(A_{\Omega}+\frac{\omega_{1}}{2 \omega}\left(A_{\Omega+\omega}-A_{\Omega-\omega}\right)\right)+\left(2 i \pi \Gamma_{0}^{2}+\Omega\right)\left(B_{\Omega}+\frac{\omega_{1}}{2 \omega}\left(B_{\Omega+\omega}-B_{\Omega-\omega}\right)\right) \\
& -2 i \pi \Gamma_{0}^{2}\left(A_{-\Omega}^{\dagger}+B_{-\Omega}^{\dagger}\right)-2 i \pi \Gamma_{0}^{2} \frac{\omega_{1}}{2 \omega}\left(A_{-\Omega+\omega}^{\dagger}+B_{-\Omega+\omega}^{\dagger}-A_{-\Omega-\omega}^{\dagger}-B_{-\Omega-\omega}^{\dagger}\right)=0, \\
& \left(2 i \pi \Gamma_{0}^{2}-\Omega\right)\left(A_{-\Omega}^{\dagger}+\frac{\omega_{1}}{2 \omega}\left(A_{-\Omega+\omega}^{\dagger}-A_{-\Omega-\omega}^{\dagger}\right)\right)+\left(2 i \pi \Gamma_{0}^{2}+\Omega\right)\left(B_{-\Omega}^{\dagger}+\frac{\omega_{1}}{2 \omega}\left(B_{-\Omega+\omega}^{\dagger}-B_{-\Omega-\omega}^{\dagger}\right)\right) \\
& -2 i \pi \Gamma_{0}^{2}\left(A_{\Omega}+B_{\Omega}\right)-2 i \pi \Gamma_{0}^{2} \frac{\omega_{1}}{2 \omega}\left(A_{\Omega+\omega}+B_{\Omega+\omega}-A_{\Omega-\omega}-B_{\Omega-\omega}\right)=0 .
\end{aligned}
$$


In order to solve this system of equations, lowest order solutions $\left(O\left(\omega_{1}^{0}\right)\right)$

$$
\begin{aligned}
B_{\Omega}^{(0)} & =\lambda_{\Omega} A_{\Omega}+\left(1-\lambda_{\Omega}\right) A_{-\Omega}^{\dagger}, \\
B_{-\Omega}^{(0) \dagger} & =\left(1-\lambda_{\Omega}\right) A_{\Omega}+\lambda_{\Omega} A_{-\Omega}^{\dagger},
\end{aligned}
$$

are substituted for $B_{\Omega \pm \omega}$. Here $\lambda_{\Omega}=\Omega /\left(\Omega+4 i \pi \Gamma_{0}^{2}\right)$. The final results to order $O\left(\omega_{1}\right)$ are:

$$
\begin{aligned}
B_{\Omega}= & \lambda_{\Omega} A_{\Omega}+\left(1-\lambda_{\Omega}\right) A_{-\Omega}^{\dagger} \\
& +\frac{\omega_{1}}{2 \omega}\left[\left(\lambda_{\Omega}-\lambda_{\Omega+\omega}\right) A_{\Omega+\omega}-\left(\lambda_{\Omega}-\lambda_{\Omega-\omega}\right) A_{\Omega-\omega}-\left(2-\lambda_{\Omega}-\lambda_{\Omega+\omega}\right) A_{-\Omega-\omega}^{\dagger}+\left(2-\lambda_{\Omega}-\lambda_{\Omega-\omega}\right) A_{-\Omega+\omega}^{\dagger}\right], \\
B_{-\Omega}^{\dagger}= & \left(1-\lambda_{\Omega}\right) A_{\Omega}+\lambda_{\Omega} A_{-\Omega}^{\dagger} \\
& +\frac{\omega_{1}}{2 \omega}\left[\left(2-\lambda_{\Omega}-\lambda_{\Omega+\omega}\right) A_{\Omega+\omega}-\left(2-\lambda_{\Omega}-\lambda_{\Omega-\omega}\right) A_{\Omega-\omega}-\left(\lambda_{\Omega}-\lambda_{\Omega+\omega}\right) A_{-\Omega-\omega}^{\dagger}+\left(\lambda_{\Omega}-\lambda_{\Omega-\omega}\right) A_{-\Omega+\omega}^{\dagger}\right] .
\end{aligned}
$$

The average backscattering current of Eq. (45) is computed using the correlator:

$$
\left\langle A_{\Omega}^{\dagger} A_{\tilde{\Omega}}\right\rangle=n_{\Omega} \delta_{\Omega, \tilde{\Omega}}
$$

where $n_{\Omega}$ is the Fermi distribution function at zero temperature, with a chemical potential $\omega_{0}$. The correlator $\left\langle B_{-\Omega}^{\dagger} B_{\tilde{\Omega}}\right\rangle$ is computed using Eqs. (B7), (B8) and the limit $x_{+}=x_{-}=0$ is specified to compute the backscattering current:

$$
\begin{aligned}
\left\langle I_{B}(t)\right\rangle= & \frac{1}{2} \int_{-\infty}^{+\infty} n_{\Omega} d \Omega\left(2-\lambda_{\Omega}-\lambda_{-\Omega}\right)-\frac{1}{2} \int_{-\infty}^{+\infty} d \Omega\left(1-\lambda_{\Omega}\right)\left(1-\lambda_{-\Omega}\right) \\
& +\frac{\omega_{1}}{4 \omega} \int_{-\infty}^{+\infty} n_{\Omega} d \Omega\left[e^{i \omega t}\left(\lambda_{\Omega}-\lambda_{-\Omega}+\lambda_{-\Omega-\omega}-\lambda_{\Omega-\omega}\right)-e^{-i \omega t}\left(\lambda_{\Omega}-\lambda_{-\Omega}+\lambda_{-} \Omega+\omega-\lambda_{\Omega+\omega}\right)\right]
\end{aligned}
$$

which then leads to Eq. (46).

1 M. Reznikov, M. Heiblum, H. Shtrikman, and D. Mahalu, Phys. Rev. Lett. 75, 3340 (1995).

2 A. Kumar, L. Saminadayar, D. C. Glattli, Y. Jin, and B. Etienne, ibid. 76, 2778 (1996).

${ }^{3}$ G. B. Lesovik, JETP Lett. 70, 208 (1999).

4 M. Büttiker, Phys. Rev. Lett. 65, 2901 (1990); Phys. Rev. B 45, 3807 (1992).

${ }^{5}$ C.W.J. Beenakker and H. van Houten, Phys. Rev. B 43, 12066 (1991).

6 T. Martin and R. Landauer, Phys. Rev. B 45, 1742 (1992).

7 C. L. Kane and M. P. A. Fisher, Phys. Rev. Lett. 72, 724 (1994).

8 C. de C. Chamon, D. E. Freed, and X. G. Wen, Phys. Rev. B 51, 2363 (1995).

9 P. Fendley, A. W. W. Ludwig, and H. Saleur, Phys. Rev. Lett. 75, 2196 (1995).

10 L. Saminadayar, D. C. Glattli, Y. Jin, and B. Etienne, Phys. Rev. Lett. 79, 2526 (1997).

11 R. de-Picciotto, M. Reznikov, M. Heiblum, V. Umansky, G. Bunin, and D. Mahalu, Nature 389, 162 (1997).

12 A. Crépieux, R. Guyon, P. Devillard, and T. Martin, Phys. Rev. B 67, 205408 (2003).

13 L. P. Kouwenhoven, S. Jauhar, J. Orenstein, P. L. McEuen, Y. Nagamune, J. Motohisa, and H. Sakaki, Phys. Rev. Lett. 73, 3443 (1994); ; L. P. Kouwenhoven, S. Jauhar, K. McCormick, D. Dixon, P. L. McEuen, Yu. V. Nazarov, N. C. van der Vaart, and C. T. Foxon, Phys. Rev. B 50, 2019 (1994); T. H. Oosterkamp, L. P. Kouwenhoven, A. E. A. Koolen, N. C. van der Vaart, and C. J. P. M. Harmans, Phys. Rev. Lett. 78, 1536 (1997).

14 C. Bruder and H. Schoeller, Phys. Rev. Lett. 72, 1076 (1994).

15 G. B. Lesovik and L. S. Levitov, Phys. Rev. Lett. 72, 538 (1994).

16 G. B. Lesovik, T. Martin, and J. Torrès, Phys. Rev. B 60, 11935 (1999); J. Torrès, T. Martin, and G. B. Lesovik, Phys. Rev. B 63, 134517 (2001).

17 S. R. Yang, Sol. State Commun. 81, 375 (1992).

18 R. J. Schoelkopf, P. J. Burke, A. A. Kozhevnikov, D. E. Prober, and M. J. Rooks, Phys. Rev. Lett. 78, 3370 (1997).

19 M. Büttiker and R. Landauer, Phys. Rev. Lett. 49, 1739 (1982).

${ }^{20}$ R. J. Schoelkopf, A. A. Kozhevnikov, D. E. Prober, and M. J. Rooks, Phys. Rev. Lett. 80, 2437 (1998).

21 Y. Levinson and P. Wölfle, Phys. Rev. Lett. 83, 1399 (1999). 
${ }^{22}$ L.H. Reydellet, P. Roche, D.C. Glattli, B. Etienne, and Y. Jin, Phys. Rev. Lett. 90, 176803 (2003).

23 M.H. Pedersen and M. Büttiker, Phys. Rev. B 58, 12993 (1998).

24 A. A. Kozhevnikov, R. J. Shoelkopf, and D. E. Prober, Phys. Rev. Lett. 84, 3398 (2000).

25 X. Jehl, M. Sanquer, R. Calemczuk, and D. Mailly, Nature 405, 50 (2000).

26 T. Martin, Phys. Lett. A 220, 137 (1996).

27 M.P.A. Fisher and L. I. Glazman in Mesoscopic Electron Transport, L. Kouwenhoven, G. Schön, and L. Sohn eds., NATO ASI Series E345, Kluwer Ac. Publ., Dordrecht (1997).

28 M. Sassetti and B. Kramer, Phys. Rev. B 54, 5203 (1996). G. Cuniberti, M. Sassetti, and B. Kramer, Phys. Rev. B 57, 1515 (1998).

29 D. Feldman and Y. Gefen, Phys. Rev. B 67, 115337 (2003).

30 C.de C. Chamon, D. E. Freed, and X. G. Wen, Phys. Rev. B 53, 4033 (1996).

31 H.H. Lin and M.P.A. Fisher, Phys. Rev. B 54, 10593 (1996).

32 C. L. Kane and M. P. A. Fisher, Phys. Rev. Lett. 68, 1220 (1992); C. L. Kane and M. P. A. Fisher, Phys. Rev. B 46, 15233 (1992).

33 X.G. Wen, Int. J. Mod. Phys. B 6, 1711 (1992); Adv. Phys. 44, 405 (1995).

34 I. Safi, P. Devillard, and T. Martin, Phys. Rev. Lett. 86, 4628 (2001).

35 C. de C. Chamon and D. E. Freed, Phys. Rev. B 60, 1842 (1999).

${ }^{36}$ G. B. Lesovik and R. Loosen, Pis'ma Zh. Eksp. Theor. Fiz. 65, 280 (1997) [JETP Lett. 65, 295 (1997)].

37 Y. Gavish, Y. Levinson, and Y. Imry, Phys. Rev. B 62, 10637 (2000); Phys. Rev. Lett. 87, 216807 (2001).

38 V. Rodriguez, P. Roche, D. C. Glattli, Y. Jin, and B. Etienne, Physica E 12, 88 (2002).

39 T. Martin and D. Loss, Int. J. Mod. Phys. B 9, 485 (1995).

40 T. Martin, Physica D 83, 216 (1995).

41 Y. Oreg and A. Finkel'stein, Phys. Rev. Lett. 74, 3668 (1995).

42 V.V. Ponomarenko, Phys. Rev. B 54, 10328 (1996).

${ }^{4}$ K. I. Imura and N. Nagaosa, Phys. Rev. B 55, 7690 (1997); K. I. Imura and N. Nagaosa, Phys. Rev. B 57, 6826 (1998). 